Research Paper

\title{
Decreased Overall and Cancer-Specific Mortality with Neoadjuvant Chemotherapy in Locoregionally Advanced Nasopharyngeal Carcinoma Treated by Intensity-modulated Radiotherapy: Multivariate Competing Risk Analysis
}

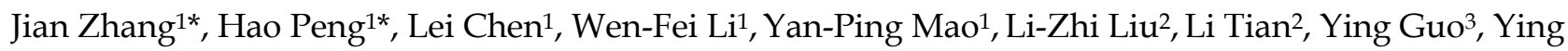
Sun $^{1}$, Jun $\mathrm{Ma}^{1 凶}$

1. Department of Radiation Oncology, Sun Yat-sen University Cancer Center, State Key Laboratory of Oncology in Southern China, Collaborative Innovation Center for Cancer Medicine, People's Republic of China;

2. Imaging Diagnosis and Interventional Center, Sun Yat-sen University Cancer Center, State Key Laboratory of Oncology in Southern China, Collaborative Innovation Center for Cancer Medicine, People's Republic of China;

3. Department of Clinical Trials Center, Sun Yat-sen University Cancer Center, State Key Laboratory of Oncology in Southern China, Collaborative Innovation Center for Cancer Medicine, People's Republic of China.

* These authors contributed equally to this work.

$\square$ Corresponding author: Jun Ma, MD, State Key Laboratory of Oncology in South China, Department of Radiation Oncology, Sun Yat-sen University Cancer Center, 651 Dongfeng Road East, Guangzhou 510060, People's Republic of China Telephone: +86-20-87343469 Fax: +86-20-87343295 E-mail: majun2@mail.sysu.edu.cn

(C) Ivyspring International Publisher. This is an open access article distributed under the terms of the Creative Commons Attribution (CC BY-NC) license (https://creativecommons.org/licenses/by-nc/4.0/). See http://ivyspring.com/terms for full terms and conditions.

Received: 2017.03.15; Accepted: 2017.07.10; Published: 2017.08.03

\begin{abstract}
Background: Value of neoadjuvant chemotherapy (NACT) is still controversial in locoregionally advanced nasopharyngeal carcinoma (LA-NPC). Based on competing risk analysis model, we aim at evaluating the efficacy of NACT in decreasing cancer-specific mortality for LA-NPC (except T3-4N0) treated by intensity-modulated radiotherapy (IMRT).

Methods: Data on 957 patients with LA-NPC were retrospectively reviewed. The cumulative incidence of cancer-specific and non-cancer-specific (competing) mortality was determined by univariate and multivariate competing risk analysis.
\end{abstract}

Results: 542 (56.6\%) patients received NACT using docetaxel with cisplatin (TP) or fluorouracil with cisplatin (PF) regimens. The median follow-up duration was 57.23 months (range, 1.27-78.53 months). In total, 161/957 (16.8\%) patients died, with 140 cancer-specific and 21 non-cancer-specific deaths were observed, respectively. In univariate analysis, the 3- and 5-year cumulative cancer-specific mortality rates for NACT vs. non-NACT group were $8.58 \%$ vs. $7.32 \%$ and $14.74 \%$ vs. $14.52 \%(P=0.95)$, respectively. With regard to competing mortality, the 3 - and 5 -year cumulative rates $(0.93 \%$ vs. $1.22 \%$ and $1.31 \%$ vs. $3.06 \%$; $P=0.196)$ were comparable between the two groups. Multivariate competing risk analysis established NACT as an independent prognostic factor in decreasing cancer-specific mortality $(\mathrm{HR}, 0.681 ; 95 \% \mathrm{Cl}, 0.488-0.951 ; P=$ $0.016)$ and overall mortality $(\mathrm{HR}, 0.654 ; 95 \% \mathrm{Cl}, 0.471-0.909 ; P=0.011)$.

Conclusions: NACT may be a powerful approach in decreasing cancer-specific mortality and overall mortality in LA-NPC treated by IMRT, and our findings would strengthen the role of NACT.

Key words: Nasopharyngeal carcinoma; competing risk analysis; neoadjuvant chemotherapy; cancer-specific mortality; competing mortality; intensity-modulated radiotherapy. 


\section{Introduction}

As a special kind of head and neck cancer, nasopharyngeal carcinoma (NPC) has an extremely unbalanced geographic distribution. Worldwide, 86,500 cases of NPC were reported in 2012 and $71 \%$ of new cases were in east and southeast parts of Asia including south China [1]. Due to the anatomic constrains, surgery is not readily accessible for NPC. Therefore, radiotherapy (RT) has been the unique and curative treatment for NPC as a result of radiation sensitivity. NPC is also sensitive to chemotherapy, and incorporation of standard RT with chemotherapy could achieve better therapeutic outcomes for patients with advanced NPC compared with RT alone [2-6]. With the advent of intensity-modulated radiotherapy (IMRT), distant metastasis has emerged as the predominant treatment failure for patients with advanced NPC [7-9]. Thus, there has been a renewed interest in the re-exploration of neoadjuvant chemotherapy (NACT) [10-16] as it may reduce distant metastasis and improve overall survival. Regretfully, no phase III trial has achieved an overall survival benefit except our recent study using neoadjuvant docetaxel plus cisplatin with fluorouracil (TPF) [17]. Therefore, value of other regimens in advanced NPC still needs to be characterized.

There may be four kinds of final clinical outcomes for patients with malignant tumor after treatment: cancer-specific mortality, non-cancerspecific mortality, survival or lost to follow-up. This could also apply to NPC. In practice, medical researches may find interest in nature and time of a particular event, cancer-specific mortality for example, and the other events are considered in competition with the event of interest. In this case, Kaplan-Meier method may be inappropriate because it treats competing events as independent censorings and overestimates the proportion of cancer-specific death. Therefore, the cumulative incidence function (CIF) [18] could be used since it takes into account the informative nature of the censoring and corresponds to the probability of occurrence of a particular event without the assumption of independence between event types.

The competing risk analysis has been widely used in cancer research like breast cancer [19], ovarian cancer [20], brain metastasis cancer [21] and kidney cancer [22]. However, to the best of our knowledge, no relative study about NPC has been reported. Given the truth that we still lack strong evidence of NACT in prolonging overall survival, we conducted this study to elucidate whether NACT using less effective regimens could decrease cancer-specific mortality or not based on competing risk analysis in locoregionally advanced NPC (LA-NPC).

\section{Materials and Methods}

\section{Patient Selection}

We retrospectively reviewed data on patients with newly diagnosed stage I-IVB NPC who were treated at Sun Yat-sen university cancer center between November 2009 and March 2012. The including criteria for this study were as follows: (1) World Health Organization (WHO) pathology type II/III; (2) stage III-IVB NPC (except T3-4N0); (3) with the data of pre-treatment Epstein-Barr virus (EBV) DNA (pre-DNA); (4) age 18 years or older. This study was approved by the Research Ethics Committee of Sun Yat-sen university cancer center. Informed consent was obtained from all the patients before treatment.

\section{Clinical Staging workup}

Prior to treatment, the medial history of patients were completed. Clinical examinations of the head and neck region, direct fibre-optic nasopharyngoscopy, magnetic resonance imaging (MRI), chest radiography, whole-body bone scan and abdominal sonography were conventionally performed. Positron emission tomography (PET)-CT was also carried out if clinically indicated. Tumour-related markers like pre-DNA were quantified. All patients received a dental evaluation before RT.

All patients were staged according to the $7^{\text {th }}$ edition of the International Union against Cancer/American Joint Committee on Cancer (UICC/AJCC) system [23]. All radiographic materials and clinical records were reviewed to minimize heterogeneity in restaging. Two radiologists (L.Z.L. and L.T.) employed at our hospital separately evaluated all of the scans and disagreements were resolved by consensus.

\section{Real-time quantitative EBV DNA PCR}

Plasma EBV DNA load was measured before treatment and plasma DNA was extracted and assayed using real-time quantitative PCR which was described previously [24]. Briefly, the real-time quantitative PCR system targeted the BamHI-W region of the EBV genome using primers 5'-GCCAGAGGTAAGTGGACTTT-3' and 5'-TACC ACCTCCTCTTCTTGCT-3'. The dual fluorescencelabelled oligomer $5^{\prime}$-(FAM) CACACCCAGGCA CACACTACACAT (TAMRA)-3' served as a probe. 
Sequence data for the EBV genome were obtained from the GeneBank sequence database.

\section{Treatment}

All the patients received IMRT at Sun Yat-sen university cancer center. The prescribed doses were 66-72Gy at 2.12-2.43 Gy/fraction to the planning target volume (PTV) of the primary gross tumour volume (GTVnx), 66-70Gy to the PTV of the GTV of the positive lymph nodes (GTVnd), 60-63Gy to the PTV of the high-risk clinical target volume (CTV1), and 54-56Gy to the PTV of the low-risk clinical target volume (CTV2). All targets were treated simultaneously using the simultaneous integrated boost technique. NACT was mainly performed for patients with advanced N (N2-3) or T4 category disease to reduce micrometastasis and shrink tumor volume, and consisted of cisplatin $\left(80 \mathrm{mg} / \mathrm{m}^{2}\right)$ with 5-fluorouracil $\left(750-1000 \mathrm{mg} / \mathrm{m}^{2}\right)(\mathrm{PF})$, docetaxel $(75$ $\left.\mathrm{mg} / \mathrm{m}^{2}\right)$ with cisplatin $\left(75 \mathrm{mg} / \mathrm{m}^{2}\right)(\mathrm{TP})$ every three weeks for two or more cycles. However, NACT would also be considered if patients had to wait a long interval before radiotherapy. Concurrent chemotherapy was cisplatin weekly $\left(40 \mathrm{mg} / \mathrm{m}^{2}\right)$ for at least $4-7$ cycles or on weeks 1,4 and $7\left(80-100 \mathrm{mg} / \mathrm{m}^{2}\right)$ of radiotherapy. Treatment was reduced or stopped in cases of unacceptable toxicity or at the patient's request.

\section{Follow-Up and Statistical Analysis}

Follow-up was measured from first day of therapy to last examination or death. Patients were followed by MRI and plasma EBV DNA at least every 3 months during first 2 years, then every 6 months thereafter (or until death). The only end point (time to first defining event) was overall survival (OS), including cancer-specific death, non-cancer-specific death, survival or lost to follow-up. We treated survival and lost to follow-up patients as censored data. Therefore, there were three kind of survival status in analysis: cancer-specific mortality, non-cancer-specific mortality (i.e. competing mortality) and censored data.

The Chi-square or non-parametric test was used to compare clinical characteristics between NACT and non-NACT groups. Factors entered into analysis included age (continuous variable), gender, lactate dehydrogenase (LDH) (continuous variable), NACT, $\mathrm{T}$ category, $\mathrm{N}$ category, overall stage, smoking, drinking and pre-DNA (continuous variable). The cumulative incidence of cancer-specific mortality and competing mortality was determined by univariate and multivariate competing risk analysis. Cox proportional hazards models for competing risks according to Fine and Gray [25] were used to study combined effects of the variables on overall mortality, cancer-specific mortality and competing mortality. The analysis was performed under the $\mathrm{R}$ 3.3.0 software. Significance was set at $P<0.05$ (2-sided).

\section{Results}

\section{Patient Characteristics}

In total, 957/1811 (52.8\%) consecutive patients meeting the criteria were recruited for this study. Of the whole cohort, the male (721)-to-female (236) ratio was 3.1, and the median age was 45 years (range, 18-78 years). NACT was delivered to 542 (56.6\%) patients, with $444(82.0 \%)$ patients receiving TP regimen and $98(18 \%)$ patients receiving PF regimen. Additionally, 159/957 (16.6\%) patients did not receive concurrent chemotherapy (CRT), of whom 113/159 $(71.1 \%)$ received NACT. The median cumulative cisplatin dose during CCRT for non-NACT and NACT groups were $200 \mathrm{mg} / \mathrm{m}^{2}$ (range, 0-320) and 200 $\mathrm{mg} / \mathrm{m}^{2}$ (range, $\left.0-300\right)$, respectively $(P=0.152)$. The omitting of chemotherapy for the other 46/159 $(28.9 \%)$ patients in non-NACT group was mainly attributed to the comorbidities like liver or kidney disease (32 of 46, 69.6\%). Moreover, 14/46 (30.4\%) patients with age more than 65 years were given RT alone according to clinicians' decision. The baseline characteristics were summarized in Table 1. Obviously, patients receiving NACT had higher percentages of advanced disease (T3-4, N3 and stage IV) and higher pre-DNA level than that of patients not receiving NACT.

\section{Survival data}

Up to the last follow-up (April 2, 2016), 77/957 $(8.0 \%)$ patients were lost to follow-up. The median follow-up duration was 57.23 months (range, 1.27-78.53 months) for the entire cohort and 59.26 months (range, 28.4-78.53 months) for the survival patients. In total, 161/957 (16.8\%) patients died, with 140 cancer-specific deaths and 21 non-cancer-specific deaths were observed, respectively. With regard to non-cancer-specific death, 8/21 (38.1\%) patients died from treatment-related comorbidities, 3/21 (14.3\%) patients died from cardiovascular disease, 4/21 $(19.0 \%)$ patients died from accident and the cause of death for the other 6/21 (28.6\%) patients remained unknown. Notably, among the survival patients, $40 / 719(5.6 \%)$ patients experienced locoregional failure, 24/719 (3.3\%) patients developed distant metastasis and 9/719 (1.3\%) patients suffered both locoregional and distant failure. 
Table 1. Baseline Characteristics of 957 patients with LA-NPC (except T3-4N0).

\begin{tabular}{|c|c|c|c|}
\hline Characteristics & NACT & Non-NACT & $P$ \\
\hline & No. (\%) & No. $(\%)$ & \\
\hline Age (y, median) & $45(18-78)$ & $46(18-78)$ & $0.092^{\mathrm{a}}$ \\
\hline Gender & & & $0.723^{b}$ \\
\hline Male & 406 (74.9) & 315 (75.9) & \\
\hline Female & $136(25.1)$ & $100(24.1)$ & \\
\hline LDH (U/L, median) & 175 (100-1632) & $171(84-564)$ & $0.007 \mathrm{a}$ \\
\hline $\mathrm{T}_{\text {category }}{ }^{c}$ & & & $<0.001^{\mathrm{b}}$ \\
\hline $\mathrm{T} 1$ & $22(4.0)$ & $22(5.3)$ & \\
\hline $\mathrm{T} 2$ & $41(7.6)$ & $19(4.6)$ & \\
\hline $\mathrm{T} 3$ & $299(55.2)$ & $309(74.4)$ & \\
\hline $\mathrm{T} 4$ & $180(33.2)$ & $65(15.7)$ & \\
\hline $\mathrm{N}$ category $\mathrm{c}$ & & & $<0.001^{b}$ \\
\hline N1 & 314 (57.9) & $280(67.5)$ & \\
\hline N2 & $123(22.7)$ & $103(24.8)$ & \\
\hline N3 & $105(19.4)$ & $32(7.7)$ & \\
\hline Overall stage $^{c}$ & & & $<0.001^{\mathrm{b}}$ \\
\hline III & $279(51.5)$ & $322(77.6)$ & \\
\hline IV & $263(48.5)$ & $93(22.4)$ & \\
\hline CRT & & & $<0.001^{b}$ \\
\hline Yes & $429(79.2)$ & $369(88.9)$ & \\
\hline No & $113(20.8)$ & $46(11.1)$ & \\
\hline Smoking & & & $0.477 \mathrm{~b}$ \\
\hline Yes & $216(39.9)$ & $156(37.6)$ & \\
\hline No & $326(60.1)$ & $259(62.4)$ & \\
\hline Drinking & & & $0.682^{b}$ \\
\hline Yes & $67(12.4)$ & $55(13.3)$ & \\
\hline No & $475(87.6)$ & $360(86.7)$ & \\
\hline $\begin{array}{l}\text { Pre-DNA (copies/ml, } \\
\text { median) }\end{array}$ & $9450(0-3710000)$ & $1830(0-6710000)$ & $<0.001^{a}$ \\
\hline Mortality & & & $0.244^{\mathrm{b}}$ \\
\hline Cancer-specific & $79(89.8)$ & $61(83.6)$ & \\
\hline Non-cancer-specific & $9(10.2)$ & $12(16.4)$ & \\
\hline \multicolumn{4}{|c|}{$\begin{array}{l}\text { Abbreviations: } \mathrm{LA}-\mathrm{NPC}=\text { locoregionally advanced nasopharyngeal carcinoma; } \\
\mathrm{NACT}=\text { neoadjuvant chemotherapy; } \mathrm{LDH}=\text { lactate dehydrogenase; } \mathrm{CRT}= \\
\text { concurrent chemotherapy; Pre-DNA = pre-treatment Epstein-Barr virus DNA. } \\
\text { a } P \text {-values were calculated by Non-parametric test. } \\
\text { b } P \text {-values were calculated by Chi-square test or Fisher exact test if indicated. } \\
\text { c According to the } 7 \text { th } \text { AJCC/UICC staging system. }\end{array}$} \\
\hline
\end{tabular}

\section{Univariate competing risk analysis}

In this univariate analysis, the 3- and 5-year cumulative cancer-specific mortality rates for NACT group vs. non-NACT group were $8.58 \%$ vs. $7.32 \%$ and $14.74 \%$ vs. $14.52 \%$ ( $P=0.95$; Figure 1$)$, respectively. With regard to competing mortality, the 3- and 5-year cumulative rates $(0.93 \%$ vs. $1.22 \%$ and $1.31 \%$ vs. $3.06 \% ; P=0.196$; Figure 1 ) were comparable between the NACT and non-NACT groups. Therefore, compared with non-NACT group, the NACT group was not associated with significantly decreased 3- and 5 -year cumulative overall mortality rates $(9.51 \%$ vs. $8.53 \%$ and $16.05 \%$ vs. $17.58 \% ; P=0.86)$. Obviously, $\mathrm{N}$ category $(P<0.001)$ and overall stage $(P<0.001)$ correlates with cancer-specific mortality, but both were not associated with competing mortality $(P=$ 0.861 and 0.066, respectively; Figure 2 and Figure 3).

\section{Multivariate competing risk analysis}

Given the truth that many prognostic factors were not balanced between these two groups, a multivariate Cox proportional hazards model was performed with considering death as a competing and adjusting for host, tumor and treatment factors. After adjusting for various factors, NACT was found to be an independent prognostic factor in decreasing cancer-specific mortality (HR, 0.681; 95\% CI, $0.488-0.951 ; P=0.016)$ and overall mortality (HR, 0.654; 95\% CI, 0.471-0.909; $P=0.011$, Table 2). Intriguingly, overall stage was found to be associated with competing mortality (HR, 2.932; 95\% CI, 1.114-7.720; $P=0.029$ ).

\section{Discussion}

To the best of our knowledge, our study was the first one to apply competing risk analysis model in investigating the prognostic value of NACT in LA-NPC treated by IMRT. Based on this model, we clarified that patients with LA-NPC could benefit from neoadjuvant TP or PF regimens, and this benefit mainly originated from decreased cancer-specific mortality which would result in a decreased overall mortality. Our findings were similar to the results of a pooled data analysis of two randomized trials carried out by Chua et al. [26]. No substantial relationship between this treatment modality and competing mortality was observed. Consequently, our findings provided a new insight into understanding the value of NACT in stage III-IVB (except T3-4N0) NPC, and this may strengthen the role of NACT in clinical practice.

The outcome of univariate analysis revealed the NACT group had similar cancer-specific mortality and overall mortality rate as the non-NACT group. However, multivariate analysis showed a significant difference of both cancer-specific and overall mortality between the two groups. This was mainly attributed to that the NACT group had a higher percentage of advanced stage and higher pre-DNA load. The unbalanced distribution of tumor-related factors should have diluted any benefit of NACT for decreasing cancer-specific and overall mortality. Furthermore, overall stage was found to be an independent prognostic factor for competing mortality. One explanation is that patients with stage IV disease received more intensive treatment regimen which would result in a higher rate of treatment-related mortality. 


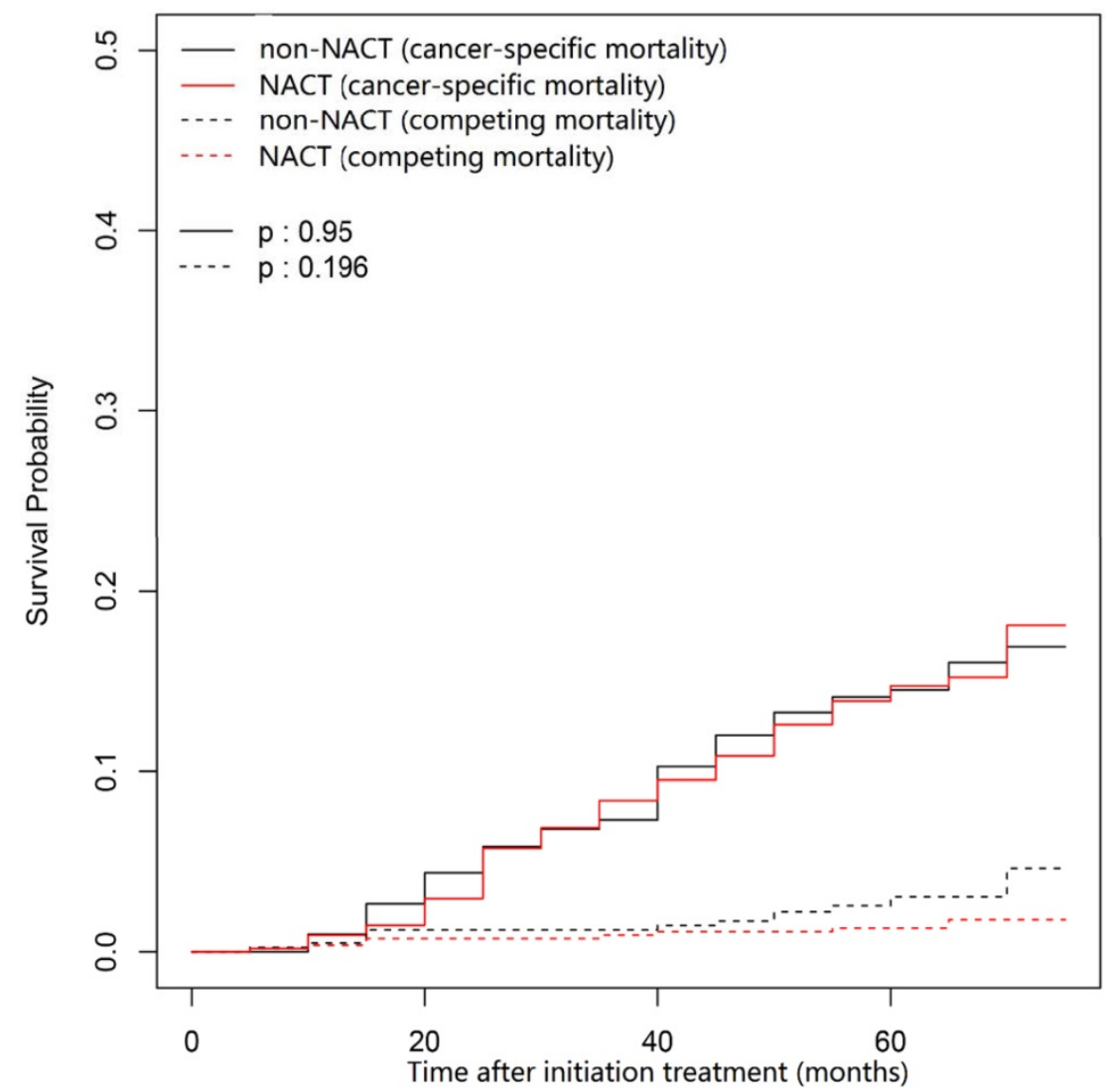

Figure 1. Cumulative cancer-specific and competing mortality curves stratified by application of NACT for the 957 patients with LA-NPC (univariate competing risk analysis). Abbreviations: NACT = neoadjuvant chemotherapy; LA-NPC = locoregionally advanced nasopharyngeal carcinoma (except T3-4N0).

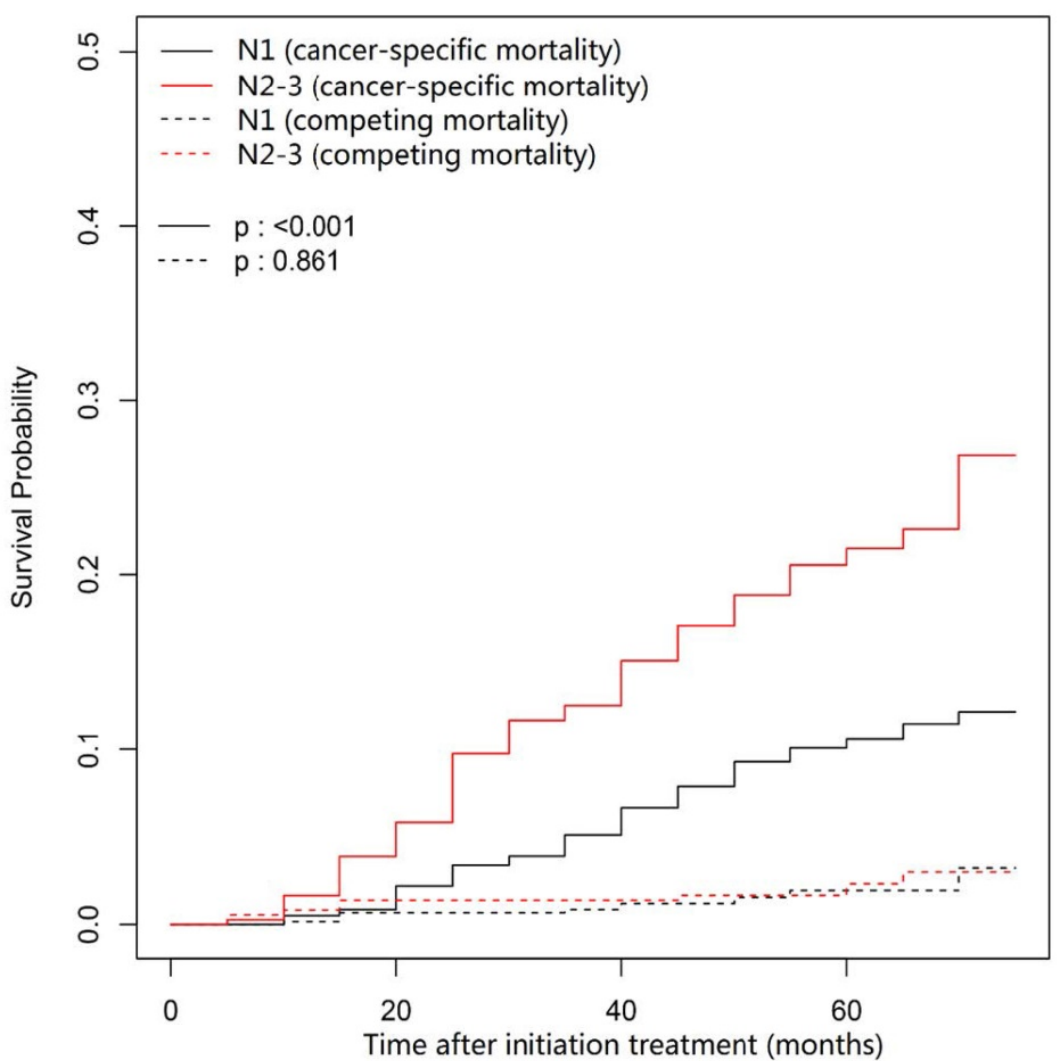

Figure 2. Cumulative cancer-specific and competing mortality curves stratified by N category (N2-3 vs. N1) for the 957 patients with LA-NPC (univariate competing risk analysis). Abbreviations: LA-NPC = locoregionally advanced nasopharyngeal carcinoma (except T3-4N0). 


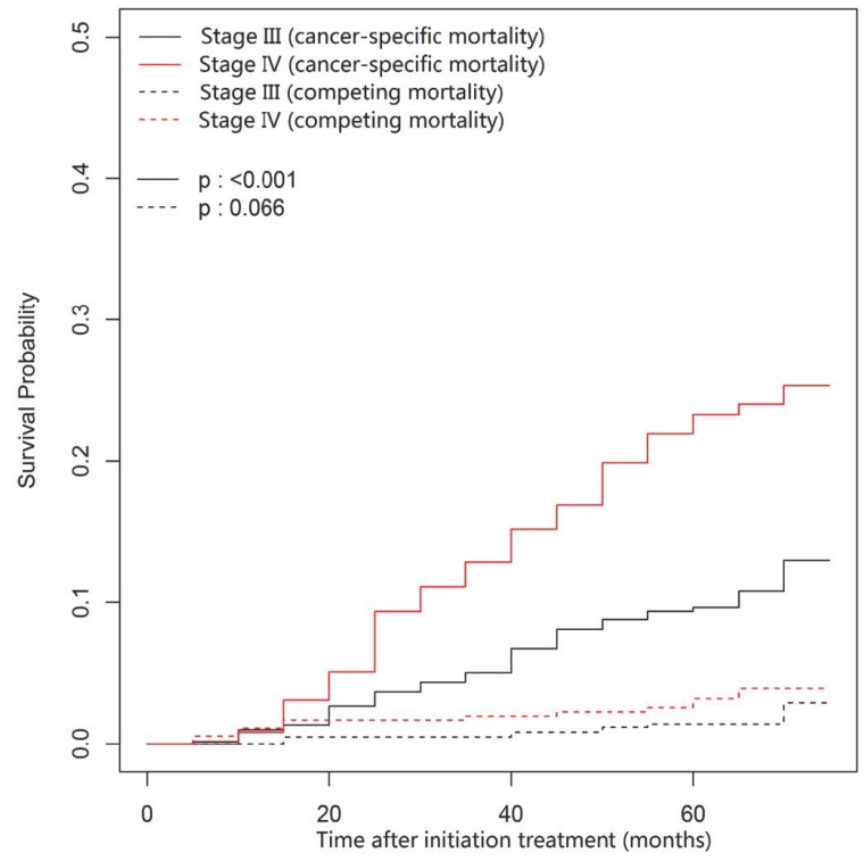

Figure 3. Cumulative cancer-specific and competing mortality curves stratified by overall stage (IV vs. III) for the 957 patients with LA-NPC (univariate competing risk analysis). Abbreviations: LA-NPC = locoregionally advanced nasopharyngeal carcinoma (except T3-4N0).

Table 2. Multivariate competing risk analysis outcomes for overall, cancer-specific and competing mortality of 957 patients with LA-NPC (except T3-4N0).

\begin{tabular}{|c|c|c|c|}
\hline Category & HR & $95 \% \mathrm{CI}$ & $P a$ \\
\hline \multicolumn{4}{|l|}{ Endpoint: overall mortality } \\
\hline Age (continuous variable, per-year increase) & 1.022 & $1.008-1.036$ & 0.0017 \\
\hline Gender (male vs. female) & 0.824 & $0.532-1.276$ & 0.39 \\
\hline LDH (continuous variable, per-U/L increase) & 1.001 & $1.000-1.003$ & 0.064 \\
\hline NACT (Yes vs. No) & 0.654 & 0.471-0.909 & 0.011 \\
\hline T category (T3-4 vs. T1-2) & 1.010 & $0.606-1.682$ & 0.97 \\
\hline N category (N2-3 vs. N1) & 1.622 & $1.131-2.328$ & 0.0086 \\
\hline Overall stage (IV vs. III) & 2.373 & $1.679-3.354$ & $<0.001$ \\
\hline Smoking (Yes vs. No) & 1.026 & $0.715-1.472$ & 0.89 \\
\hline Drinking (Yes vs. No) & 0.984 & $0.602-1.607$ & 0.95 \\
\hline Pre-DNA (continuous variable, per-copy/ml increase) & 1.000 & $1.000-1.001$ & 0.0015 \\
\hline \multicolumn{4}{|l|}{ Endpoint: cancer-specific mortality } \\
\hline Age (continuous variable, per-year increase) & 1.016 & $1.001-1.03$ & 0.032 \\
\hline Gender (male vs. female) & 0.820 & $0.514-1.310$ & 0.41 \\
\hline LDH (continuous variable, per-U/L increase) & 1.002 & $1.000-1.003$ & 0.044 \\
\hline NACT (Yes vs. No) & 0.681 & $0.488-0.951$ & 0.016 \\
\hline T category (T3-4 vs. T1-2) & 1.143 & $0.663-1.970$ & 0.63 \\
\hline N category (N2-3 vs. N1) & 1.871 & $1.274-2.750$ & 0.0014 \\
\hline Overall stage (IV vs. III) & 2.246 & $1.554-3.250$ & $<0.001$ \\
\hline Smoking (Yes vs. No) & 1.101 & $0.749-1.620$ & 0.62 \\
\hline Drinking (Yes vs. No) & 0.899 & $0.528-1.530$ & 0.70 \\
\hline Pre-DNA (continuous variable, per-copy/ml increase) & 1.000 & $1.000-1.000$ & 0.0061 \\
\hline \multicolumn{4}{|l|}{ Endpoint: competing mortality } \\
\hline Age (continuous variable, per-year increase) & 1.058 & $1.018-1.100$ & 0.0042 \\
\hline Gender (male vs. female) & 0.708 & $0.208-2.410$ & 0.58 \\
\hline LDH (continuous variable, per-U/L increase) & 1.001 & $0.999-1.001$ & 0.53 \\
\hline NACT (Yes vs. No) & 0.476 & $0.188-1.200$ & 0.12 \\
\hline T category (T3-4 vs. T1-2) & 0.450 & $0.095-2.140$ & 0.32 \\
\hline N category (N2-3 vs. N1) & 0.679 & $0.216-2.140$ & 0.51 \\
\hline Overall stage (IV vs. III) & 2.932 & $1.114-7.720$ & 0.029 \\
\hline Smoking (Yes vs. No) & 0.649 & $0.226-1.870$ & 0.42 \\
\hline Drinking (Yes vs. No) & 1.362 & $0.433-4.280$ & 0.60 \\
\hline Pre-DNA (continuous variable, per-copy/ml increase) & 0.998 & $0.992-1.002$ & 0.55 \\
\hline
\end{tabular}


Distant metastasis has been the main failure pattern after initial concurrent chemoradiotherapy (CCRT) especially when IMRT technique was applied [7-9]. Therefore, additional cycles of chemotherapy, such as adding adjuvant/neoadjuvant chemotherapy to CCRT, may improve prognosis. However, our previous study characterized that the value of adjuvant chemotherapy using PF regimen might be limited [27]. Moreover, the low rates of compliance $(52-62 \%)[2,3,27-29]$ to adjuvant chemotherapy also would constrain the utility. Thus, NACT prior to CCRT could be a promising treatment modality. Regretfully, almost all the previous randomized phase III trials focusing on NACT failed to obtain a positive result on OS except our recent study using TPF regimen [17]. The negative outcomes may be attributed to following two reasonable explanations. Firstly, most trials included LA-NPC without excluding T3-4N0 disease, and this part of patients may not have higher distant tumor burden and would not really benefit from NACT. Hence, the recruitment of these patients could narrow the benefit of NACT and therefore resulted in insignificant outcomes. Furthermore, a truly effective neoadjuvant regimen has not yet been identified. Three randomized phase trials [30-32] and a meta-analysis [33] revealed TPF was superior to PF in head and neck cancers. Most recently, our randomized trial using TPF regimen also achieved positive results in advanced NPC. These results indicated that neoadjuvant TPF regimen may be more effective. However, the value of less effective regimens (PF or TP) remains unknown if appropriate subpopulation is selected. In our current study, we clarified that less effective regimens could also achieved a significantly decreased cancer-specific and overall mortality when patients with high-risk of distant metastasis (stage III-IV except T3-4N0) were selected. Besides, a meta-analysis carried out by Ouyang et al. [34] also supported our findings. Therefore, NACT still remains a promising treatment strategy irrespective of the regimen, but the key is to select appropriate patients who could benefit from it. Another of our ongoing trials (NCT01872962) is awaiting to be reported to further confirm the value of NACT.

The main strength of this study is that pre-DNA was included in analysis. In addition to tumor stage serving as an indicating factor for performing NACT, pre-DNA also has been proven to be a reliable biomarker [35-38] in predicting prognosis. Actually, pre-DNA was also associated with significantly increased cancer-specific and overall mortality in this current study. Our previous studies [39, 40] have demonstrated that pre-DNA could define high- or low-risk patients with advanced NPC who may benefit from NACT or not. Therefore, combination of these multiple prognostic factors is a powerful method for helping delivering individualized NACT.

In conclusion, our findings suggested that NACT using TP or PF regimens could still provide significant benefit for patients with LA-NPC (except T3-4N0) in decreasing cancer-specific and overall mortality. Therefore, it is a feasible approach that leads to better prognosis in the era of IMRT. Apparently, the shortcomings of this study should also be acknowledged: this analysis was performed retrospectively at a single center. Furthermore, the NACT regimens used in this study were non-uniform. However, this may not affect the conclusions because there is no evidence showing the efficacy difference of these two regimens. Also, longer follow-up time may be needed to observe more endpoints since many patients in this study still survival with recurrent disease. Further randomized trials should be warranted to confirm the results of this study.

\section{Conclusions}

Based on this competing risk analysis model, we clarified that neoadjuvant chemotherapy using TP or PF regimens could decrease cancer-specific mortality and overall mortality for patients with LA-NPC (except T3-4N0) receiving IMRT. Our findings may further strengthen the role of NACT in LA-NPC treated by IMRT.

\section{Abbreviations}

NPC: nasopharyngeal carcinoma; RT: radiotherapy; IMRT: intensity-modulated radiotherapy; NACT: neoadjuvant chemotherapy; TPF: docetaxel plus cisplatin with fluorouracil; CIF: cumulative incidence function; LA-NPC: locoregionally advanced nasopharyngeal carcinoma; WHO: World Health Organization; EBV: Epstein-Barr virus; pre-DNA: pre-treatment Epstein-Barr virus DNA; MRI: magnetic resonance imaging; PET: Positron emission tomography; UICC/AJCC: International Union against Cancer/American Joint Committee on Cancer; PTV: planning target volume; GTVnx: primary gross tumour volume; GTVnd: gross tumour volume of the positive lymph nodes; CTV1: high-risk clinical target volume; CTV2: low-risk clinical target volume; PF: cisplatin with fluorouracil; TP: docetaxel with cisplatin; OS: overall survival; LDH: lactate dehydrogenase; CRT: concurrent chemotherapy.

\section{Acknowledgments}

This work was supported by grants from the Sun Yat-sen University Clinical Research 5010 Program (No. 2012011), the Science and Technology Project of 
Guangzhou City, China (No. 14570006), and the Planned Science and Technology Project of Guangdong Province, China (No. 2013B020400004).

\section{Competing Interests}

The authors have declared that no competing interest exists.

\section{References}

1. Torre LA, Bray F, Siegel RL, et al. Global cancer statistics, 2012. CA Cancer J Clin. 2015; 65: 87-108.

2. Wee J, Tan EH, Tai BC, et al. Randomized trial of radiotherapy versus concurrent chemoradiotherapy followed by adjuvant chemotherapy in patients with American Joint Committee on Cancer/International Union against cancer stage III and IV nasopharyngeal cancer of the endemic variety. J Clin Oncol. 2005; 23: 6730-6738.

3. Al-Sarraf M, LeBlanc M, Giri PG, et al. Chemoradiotherapy versus radiotherapy in patients with advanced nasopharyngeal cancer: phase III randomized Intergroup study 0099. J Clin Oncol. 1998; 16: 1310-1317.

4. Lee AW, Lau WH, Tung SY, et al. Preliminary results of a randomized study on therapeutic gain by concurrent chemotherapy for regionally-advanced nasopharyngeal carcinoma: NPC-9901 Trial by the Hong Kong Nasopharyngeal Cancer Study Group. J Clin Oncol. 2005; 23: 6966-6975.

5. Lin JC, Jan JS, Hsu CY, et al. Phase III study of concurrent chemoradiotherapy versus radiotherapy alone for advanced nasopharyngeal carcinoma: positive effect on overall and progression-free survival. J Clin Oncol. 2003; 21: 631-637.

6. Chan AT, Teo PM, Ngan RK, et al. Concurrent chemotherapy-radiotherapy compared with radiotherapy alone in locoregionally advanced nasopharyngeal carcinoma: progression-free survival analysis of a phase III randomized trial. J Clin Oncol. 2002; 20: 2038-2044.

7. Sun $X$, Su S, Chen C, et al. Long-term outcomes of intensity-modulated radiotherapy for 868 patients with nasopharyngeal carcinoma: an analysis of survival and treatment toxicities. Radiother Oncol. 2014; 110: 398-403.

8. Kam MK, Teo PM, Chau RM, et al. Treatment of nasopharyngeal carcinoma with intensity-modulated radiotherapy: the Hong Kong experience. Int J Radiat Oncol Biol Phys. 2004; 60: 1440-1450.

9. Lai SZ, Li WF, Chen L, et al. How does intensity-modulated radiotherapy versus conventional two-dimensional radiotherapy influence the treatment results in nasopharyngeal carcinoma patients? Int J Radiat Oncol Biol Phys. 2011: 80: 661-668.

10. Hui EP, Ma BB, Leung SF, et al. Randomized phase II trial of concurrent cisplatin-radiotherapy with or without neoadjuvant docetaxel and cisplatin in advanced nasopharyngeal carcinoma. J Clin Oncol. 2009; 27: 242-249.

11. Tan T, Lim WT, Fong KW, et al. Concurrent chemo-radiation with or without induction gemcitabine, Carboplatin, and Paclitaxel: a randomized, phase 2/3 trial in locally advanced nasopharyngeal carcinoma. Int J Radiat Oncol Biol Phys. 2015; 91: 952-960.

12. Chua DT, Sham JS, Choy D, et al. Preliminary report of the Asian-Oceanian Clinical Oncology Association randomized trial comparing cisplatin and epirubicin followed by radiotherapy versus radiotherapy alone in the treatment of patients with locoregionally advanced nasopharyngeal carcinoma. Asian-Oceanian Clinical Oncology Association Nasopharynx Cancer Study Group. Cancer. 1998; 83: 2270-2283.

13. Fountzilas $\mathrm{G}$, Ciuleanu $\mathrm{E}$, Bobos $\mathrm{M}$, et al. Induction chemotherapy followed by concomitant radiotherapy and weekly cisplatin versus the same concomitant chemoradiotherapy in patients with nasopharyngeal carcinoma: a randomized phase II study conducted by the Hellenic Cooperative Oncology Group (HeCOG) with biomarker evaluation. Ann Oncol. 2012; 23: 427-435.

14. Hareyama M, Sakata $\mathrm{K}$, Shirato $\mathrm{H}$, et al. A prospective, randomized trial comparing neoadjuvant chemotherapy with radiotherapy alone in patients with advanced nasopharyngeal carcinoma. Cancer. 2002; 94: 2217-2223.

15. Ma J, Mai HQ, Hong MH, et al. Results of a prospective randomized trial comparing neoadjuvant chemotherapy plus radiotherapy with radiotherapy alone in patients with locoregionally advanced nasopharyngeal carcinoma. J Clin Oncol. 2001; 19: 1350-1357.

16. International Nasopharynx Cancer Study G, Trial VI. Preliminary results of a randomized trial comparing neoadjuvant chemotherapy (cisplatin, epirubicin, bleomycin) plus radiotherapy vs. radiotherapy alone in stage $\operatorname{IV}(>$ or $=\mathrm{N} 2$, M0) undifferentiated nasopharyngeal carcinoma: a positive effect on progression-free survival. Int J Radiat Oncol Biol Phys. 1996; 35: 463-469.

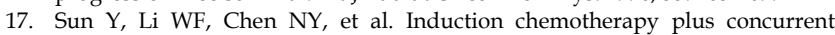
chemoradiotherapy versus concurrent chemoradiotherapy alone in locoregionally advanced nasopharyngeal carcinoma: a phase 3, multicentre, randomised controlled trial. Lancet Oncol. 2016; 17: 1509-1520.

18. Kalbfleisch JD, Prentice RL. The statistical analysis of failure time data, vol 360. Wiley, Hoboken 2011

19. Cabarrou B, Belin L, Somda SM, et al. Prediction of long-term cumulative incidences based on short-term parametric model for competing risks: application in early breast cancer. Breast Cancer Res Treat. 2016; 156: 577-585.
20. Chang $\mathrm{YH}, \mathrm{Li} \mathrm{WH}$, Chang $\mathrm{Y}$, et al. Front-line intraperitoneal versus intravenous chemotherapy in stage III-IV epithelial ovarian, tubal, and peritoneal cancer with minimal residual disease: a competing risk analysis. BMC Cancer. 2016; 16: 235

21. Lucas JT, Jr., Colmer HGt, White L, et al. Competing Risk Analysis of Neurologic versus Nonneurologic Death in Patients Undergoing Radiosurgical Salvage After Whole-Brain Radiation Therapy Failure: Who Actually Dies of Their Brain Metastases? Int J Radiat Oncol Biol Phys. 2015; 92: 1008-1015

22. Hollingsworth JM, Miller DC, Daignault S, et al. Five-year survival after surgical treatment for kidney cancer: a population-based competing risk analysis. Cancer. 2007; 109: 1763-1768.

23. Edge SB, Compton CC. The American Joint Committee on Cancer: the 7th edition of the AJCC cancer staging manual and the future of TNM. Ann Surg Oncol. 2010; 17: 1471-1474.

24. Shao JY, Zhang Y, Li YH, et al. Comparison of Epstein-Barr virus DNA level in plasma, peripheral blood cell and tumor tissue in nasopharyngeal carcinoma. Anticancer Res. 2004; 24: 4059-4066.

25. Dogan S, Hedberg ML, Ferris RL, et al. Human papillomavirus and Epstein-Barr virus in nasopharyngeal carcinoma in a low-incidence population. Head Neck. 2014; 36: 511-516.

26. Chua DT, Ma J, Sham JS, et al. Long-term survival after cisplatin-based induction chemotherapy and radiotherapy for nasopharyngeal carcinoma: a pooled data analysis of two phase III trials. J Clin Oncol. 2005; 23: 1118-1124.

27. Chen L, Hu CS, Chen XZ, et al. Concurrent chemoradiotherapy plus adjuvant chemotherapy versus concurrent chemoradiotherapy alone in patients with locoregionally advanced nasopharyngeal carcinoma: a phase 3 multicentre randomised controlled trial. Lancet Oncol. 2012; 13: 163-171.

28. Chen Y, Liu MZ, Liang SB, et al. Preliminary results of a prospective randomized trial comparing concurrent chemoradiotherapy plus adjuvant chemotherapy with radiotherapy alone in patients with locoregionally advanced nasopharyngeal carcinoma in endemic regions of china. Int J Radiat Oncol Biol Phys. 2008; 71: 1356-1364

29. Lee AW, Tung SY, Chua DT, et al. Randomized trial of radiotherapy plus concurrent-adjuvant chemotherapy vs radiotherapy alone for regionally advanced nasopharyngeal carcinoma. J Natl Cancer Inst. 2010; 102: 1188-1198.

30. Posner MR, Hershock DM, Blajman CR, et al. Cisplatin and fluorouracil alone or with docetaxel in head and neck cancer. N Engl J Med. 2007; 357: 1705-1715.

31. Vermorken JB, Remenar E, van Herpen C, et al. Cisplatin, fluorouracil, and docetaxel in unresectable head and neck cancer. N Engl J Med. 2007; 357: 1695-1704.

32. Pointreau $\mathrm{Y}$, Garaud $\mathrm{P}$, Chapet $\mathrm{S}$, et al. Randomized trial of induction chemotherapy with cisplatin and 5-fluorouracil with or without docetaxel for larynx preservation. J Natl Cancer Inst. 2009; 101: 498-506.

33. Blanchard P, Baujat $B$, Holostenco V, et al. Meta-analysis of chemotherapy in head and neck cancer (MACH-NC): a comprehensive analysis by tumour site. Radiother Oncol. 2011; 100: 33-40.

34. OuYang PY, Xie C, Mao YP, et al. Significant efficacies of neoadjuvant and adjuvant chemotherapy for nasopharyngeal carcinoma by meta-analysis of published literature-based randomized, controlled trials. Ann Oncol. 2013; 24: 2136-2146.

35. Peng H, Guo R, Chen L, et al. Prognostic Impact of Plasma Epstein-Barr Virus DNA in Patients with Nasopharyngeal Carcinoma Treated using Intensity-Modulated Radiation Therapy. Sci Rep. 2016; 6: 22000.

36. An X, Wang FH, Ding PR, et al. Plasma Epstein-Barr virus DNA level strongly predicts survival in metastatic/recurrent nasopharyngeal carcinoma treated with palliative chemotherapy. Cancer. 2011; 117: 3750-3757.

37. Lin JC, Wang WY, Chen KY, et al. Quantification of plasma Epstein-Barr virus DNA in patients with advanced nasopharyngeal carcinoma. N Engl J Med. 2004; 350: 2461-2470.

38. Lin JC, Wang WY, Liang WM, et al. Long-term prognostic effects of plasma epstein-barr virus DNA by minor groove binder-probe real-time quantitative PCR on nasopharyngeal carcinoma patients receiving concurrent chemoradiotherapy. Int J Radiat Oncol Biol Phys. 2007; 68: 1342-1348.

39. Du XJ, Tang LL, Chen $\mathrm{L}$, et al. Neoadjuvant chemotherapy in locally advanced nasopharyngeal carcinoma: Defining high-risk patients who may benefit before concurrent chemotherapy combined with intensity-modulated radiotherapy. Sci Rep. 2015; 5: 16664.

40. Peng H, Chen L, Li WF, et al. Prognostic Value of Neoadjuvant Chemotherapy in Locoregionally Advanced Nasopharyngeal Carcinoma with Low Pre-treatment Epstein-Barr Virus DNA: a Propensity-matched Analysis. J Cancer. 2016; 7: 1465-1471 